\title{
Pancreatoblastoma with Portal Venous Invasion: A Case Series
}

\author{
Pooja Abbey ${ }^{1}$ Rama Anand $^{1}$ \\ 1 Department of Radio-Diagnosis, Lady Hardinge Medical College and \\ Associated Hospitals, New Delhi, India \\ Indian J Radiol Imaging 2021;31:611-617.
}

Address for correspondence Pooja Abbey, MD, Department of RadioDiagnosis, Lady Hardinge Medical College and Associated Hospitals, New Delhi 110001, India (e-mail: pooja_abbey@yahoo.co.in).
Abstract
Keywords
- pancreatoblastoma
- computed tomography
- portal vein invasion

We describe the sonography and computed tomography (CT) findings in three children with pancreatoblastoma and highlight the tendency of these tumors to invade the splenoportal axis. All three of our cases showed tumor thrombus within the portal vein which is a helpful diagnostic feature on imaging studies.

\section{Introduction}

Pancreatic neoplasms are uncommon in children. Pancreatoblastoma is the most common tumor arising from the pancreas in children in the first decade of life and usually presents as a large, solid, abdominal mass. ${ }^{1,2}$ Identification of pancreas as the organ of origin on cross-sectional imaging is helpful in differentiation from more common pediatric abdominal tumors like neuroblastoma or Wilm's tumor.

We report the ultrasonography (USG) and computed tomography (CT) findings in three cases of pancreatoblastoma. All three cases showed presence of tumor invasion into the portal vein which was a helpful pointer to the correct diagnosis.

\section{Case Reports}

\section{Case 1}

A 2-year-old girl was brought to the emergency with bleeding per rectum and pain abdomen for the last 5 days. On examination, a lump was palpable in the left mid abdomen which was firm in consistency and nontender. The child was pale and irritable. Hemoglobin was $3.3 \mathrm{~g} / \mathrm{dL}$.
USG abdomen ( - Fig. 1) revealed a large solid mass in the mid-left abdomen which was separated from the left kidney and spleen. The mass had well-defined margins and was predominantly solid. It was iso- to hypoechoic compared with liver parenchyma and showed internal vascularity on color Doppler. Few cystic areas were seen within the mass, a larger ( 3-cm sized) cystic component was also seen along with at its peripheral aspect. No areas of calcification were visible. Scans through the liver showed lesions of similar echogenicity which were thought to be liver metastases, and a possibility of (extra adrenal) neuroblastoma was initially considered.

Contrast-enhanced CT (CECT) was then done ( - Fig. 2) for further evaluation. The CT scanogram showed an added soft tissue density mass causing inferior displacement of the transverse colon and splenic flexure, indicating its location in the supracolic compartment. A large solid mass was seen which showed mild heterogeneous enhancement and multiple nonenhancing, necrotic areas. The mass was seen to replace the body and tail of pancreas which suggested pancreatic origin. No calcification was seen. The retropancreatic splenic vein was not opacified with contrast, instead it showed presence of soft tissue showing enhancement
DOI https://doi.org/ $10.1055 / \mathrm{s}-0041-1735914$. ISSN 0971-3026. (c) 2021. Indian Radiological Association. All rights reserved. This is an open access article published by Thieme under the terms of the Creative Commons Attribution-NonDerivative-NonCommercial-License, permitting copying and reproduction so long as the original work is given appropriate credit. Contents may not be used for commercial purposes, or adapted, remixed, transformed or built upon. (https://creativecommons.org/ licenses/by-nc-nd/4.0/)

Thieme Medical and Scientific Publishers Pvt. Ltd., A-12, 2nd Floor, Sector 2, Noida-201301 UP, India 


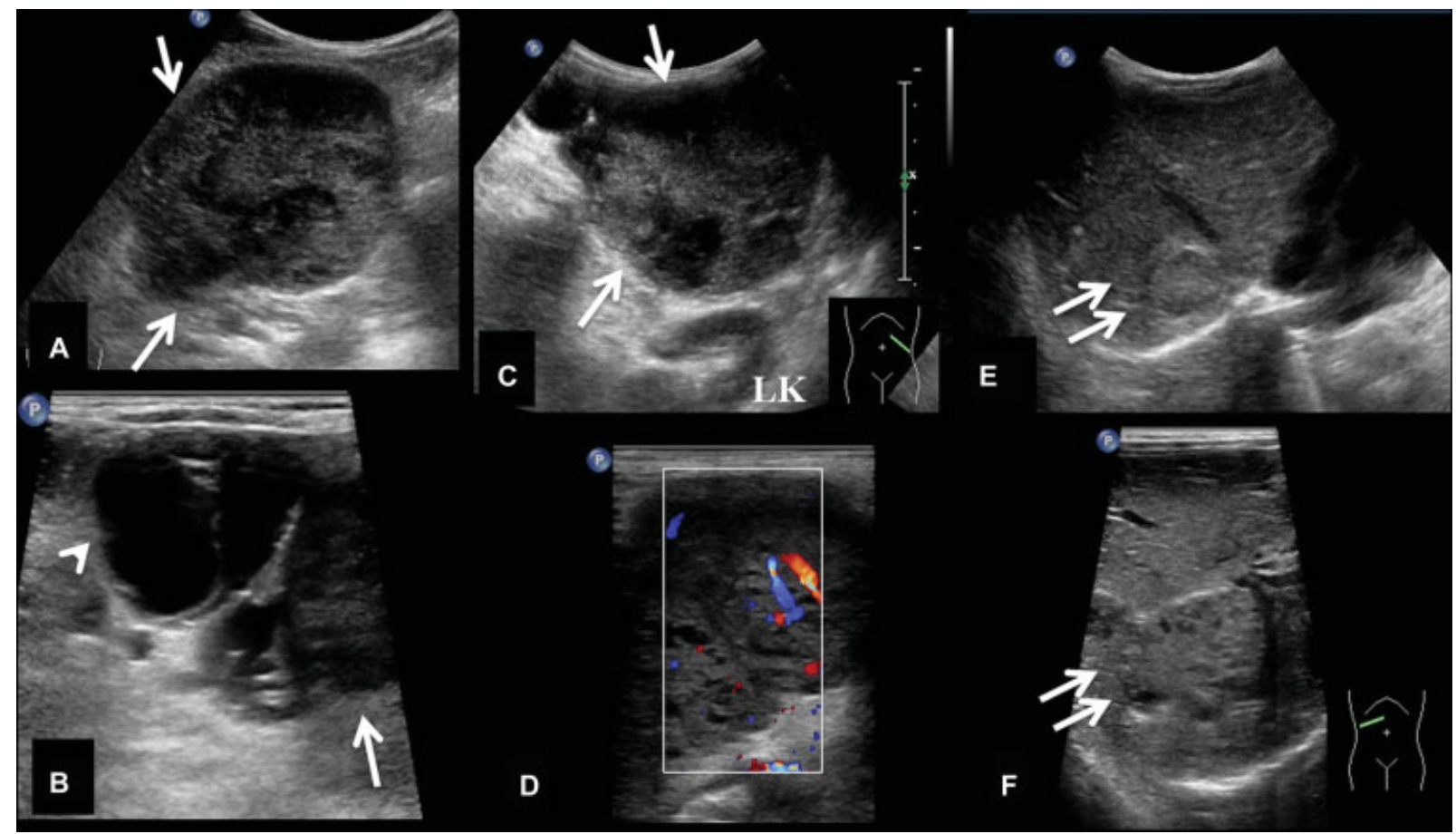

Fig. 1 Pancreatoblastoma in a 2-year-old female. USG (A-C) shows a large, circumscribed solid mass (arrows) with few cystic areas (arrowhead) in the left upper-middle abdomen, separate from the left kidney (LK). It shows internal vascularity on color Doppler (D). Scans through the liver $(\mathrm{E}, \mathrm{F})$ show similar echogenicity lesions (double arrows) which were initially thought to be liver metastases, but actually represent tumor within the portal vein branches. USG, ultrasonography.

similar to the mass. This suggested presence of intravascular tumor thrombus which could be seen extending into the portal vein and its intrahepatic branches, causing their expansion. Multiple venous collaterals were present in the periportal, gastrosplenic region, mesentery, and retroperitoneum, extending inferiorly around the rectosigmoid colon, a likely cause of the per-rectal bleeding in this child. No separate liver lesions (metastases), ascites, or significant lymph nodes were seen.

These imaging findings suggested a pancreatic mass with tumor thrombus in the splenic and portal veins. The possibility of pancreatoblastoma was considered which was confirmed on biopsy. The child was advised chemotherapy but the parents refused to consent for the same.

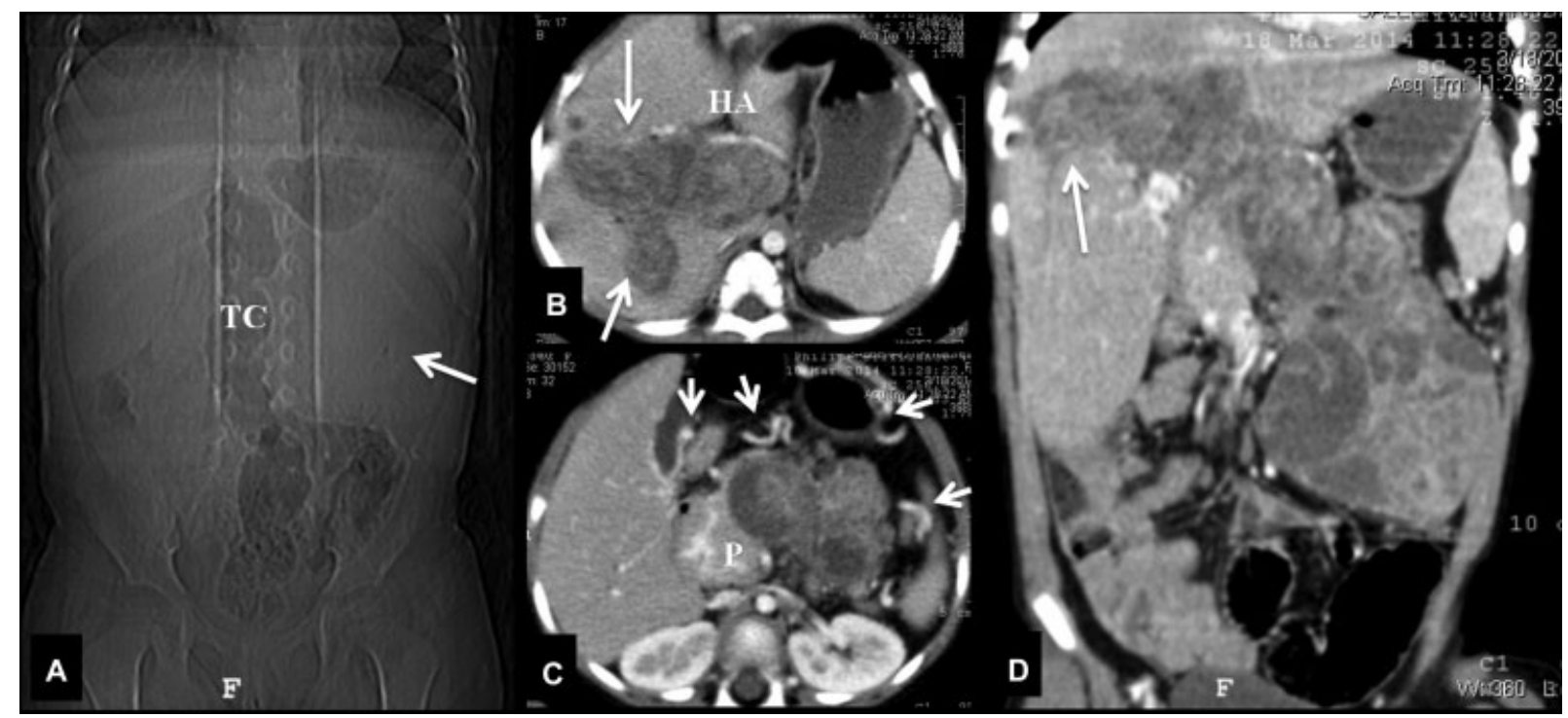

Fig. 2 Same child as in - Fig. 1. CT topogram (A) shows a soft tissue density (arrow) displacing the transverse colon (TC) inferiorly. CECT axial (B, C) and coronal (D) images reveal a large mass replacing the body and tail of pancreas, only head of pancreas is seen (P). A similar density thrombus is seen expanding the portal vein (posterior to the hepatic artery [HA]) and its intrahepatic branches (long arrows). Multiple venous collaterals are also seen (short arrows in C). There were no liver metastases. CECT, contrast-enhanced computed tomography. 


\section{Case 2}

A 7-month-old male baby presented with lump in the left upper abdomen for 4 weeks with increase in size over the last week. The mother gave a history of excessive crying and restlessness. A large lump was palpable in the left hypochondrium: firm, nontender, and not moving with respiration.

USG abdomen ( - Fig. $\mathbf{3}$ ) revealed a large solid mass in the left upper and middle abdomen. It was separate from the liver and kidneys, lying anterior to the vertebral column, suggesting a possible retroperitoneal location. The mass was predominantly hyperechoic (compared with liver) and showed slight heterogeneity with no definite calcification. It showed loss of fat planes with the liver and porta hepatis, suggesting a malignant mass.

CECT (-Fig. 4) of the infant showed a heterogeneously enhancing solid mass with large necrotic areas, no fat density or calcification seen in the region of lesser sac, with anterior displacement of stomach. There was loss of fat plans with the greater curvature of stomach. A positive "claw" sign was seen with the proximal pancreatic body which suggested origin from the pancreas. The pancreatic head was seen separately and appeared normal, but the pancreatic body and tail were not seen separate from the mass. Multiple collaterals were seen around the mass. There was nonopacification of the splenic vein and main portal vein which showed thrombus of the same density as the mass lesion. This tumor thrombus was seen extending into the right branch of portal vein and proximal left portal vein as well. Superior mesenteric vein showed normal opacification with contrast, and venous collaterals were seen draining into it.

The child showed elevated levels of $\alpha$ fetoprotein $(>2,000$ $\mathrm{ng} / \mathrm{mL}$ ), due to which a differential diagnosis of germ cell tumor was also considered. However, USG of the scrotum revealed normal bilateral testes; and the positive "claw" sign with pancreas seen on CT strongly suggested a mass of pancreatic origin rather than a retroperitoneal germ cell tumor. The diagnosis of pancreatoblastoma was confirmed by biopsy, and the child was started on chemotherapy.

\section{Case 3}

An 8-year-old boy presented with constipation and abdominal lump for o1 ne month. Examination revealed a palpable lump in the epigastrium, along with pallor. Hemoglobin was $4 \mathrm{~g} / \mathrm{dL}$; serum amylase was normal but lipase was elevated (312 U/L).

USG abdomen (-Fig. 5) revealed a solid mass, heterogeneously hyperechoic, involving the pancreatic body. There was intraluminal thrombus seen within the splenoportal axis and intrahepatic portal vein which showed an appearance similar to the mass.

CT confirmed these findings. Limited noncontrast sections showed few areas of calcification within the mass. Origin of the mass was from the distal body and tail of pancreas. It showed heterogeneous enhancement postcontrast. There was encasement of the splenic artery. The extent of intravascular tumor thrombus was well delineated on CT,

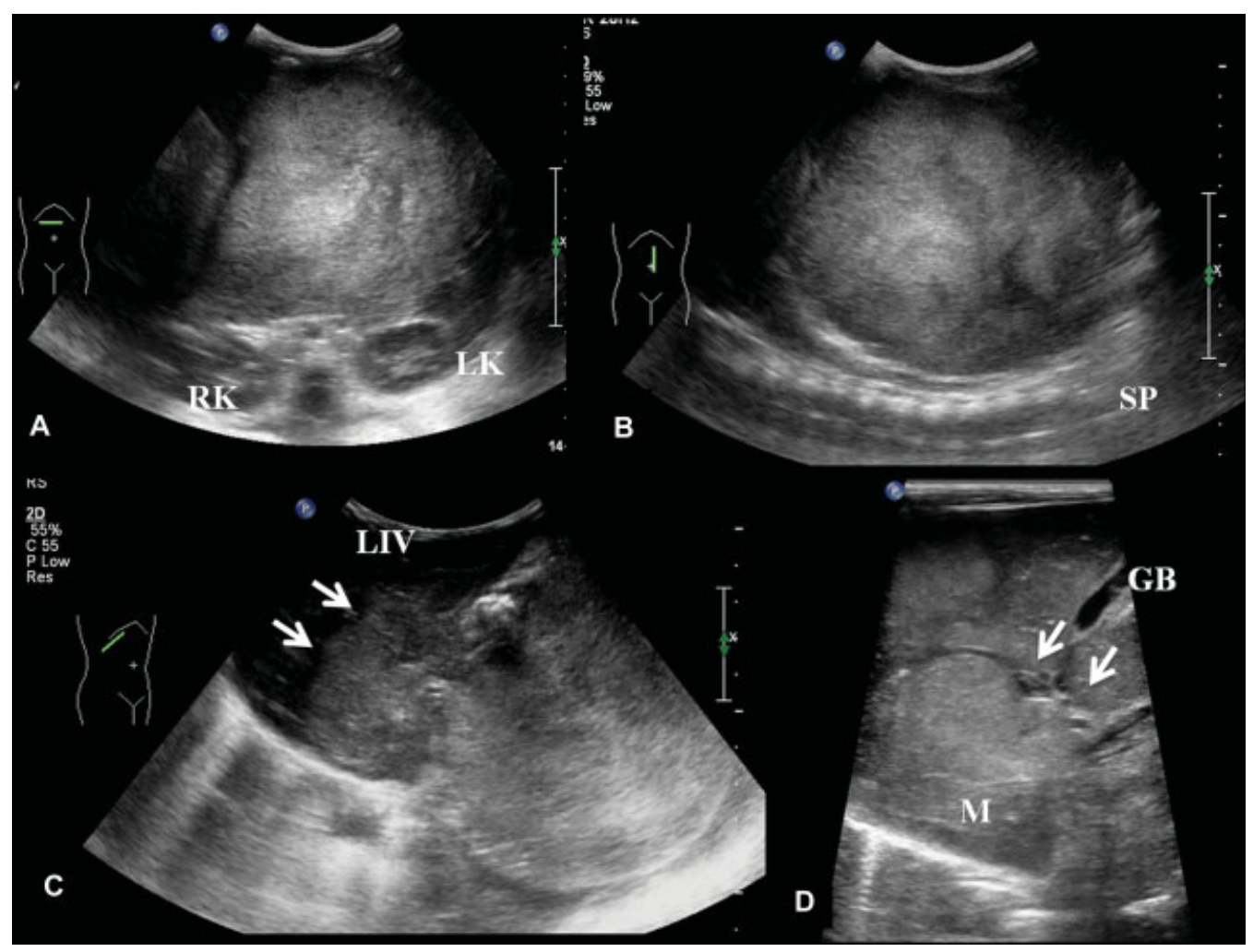

Fig. 3 A 7-month-old male with pancreatoblastoma. Ultrasonography (USG) transverse (A) and longitudinal (B) images show a large, heterogeneous hyperechoic mass in the upper abdomen, separated from the kidneys (right kidney [RK] and left kidney[LK]), and anterior to the spine (SP). On right anterior oblique scans (C, D), the mass (M) reaches uptill the liver (LIV) and porta hepatis, with loss of fat planes (arrows). Definite portal vein invasion could not be diagnosed on USG. 


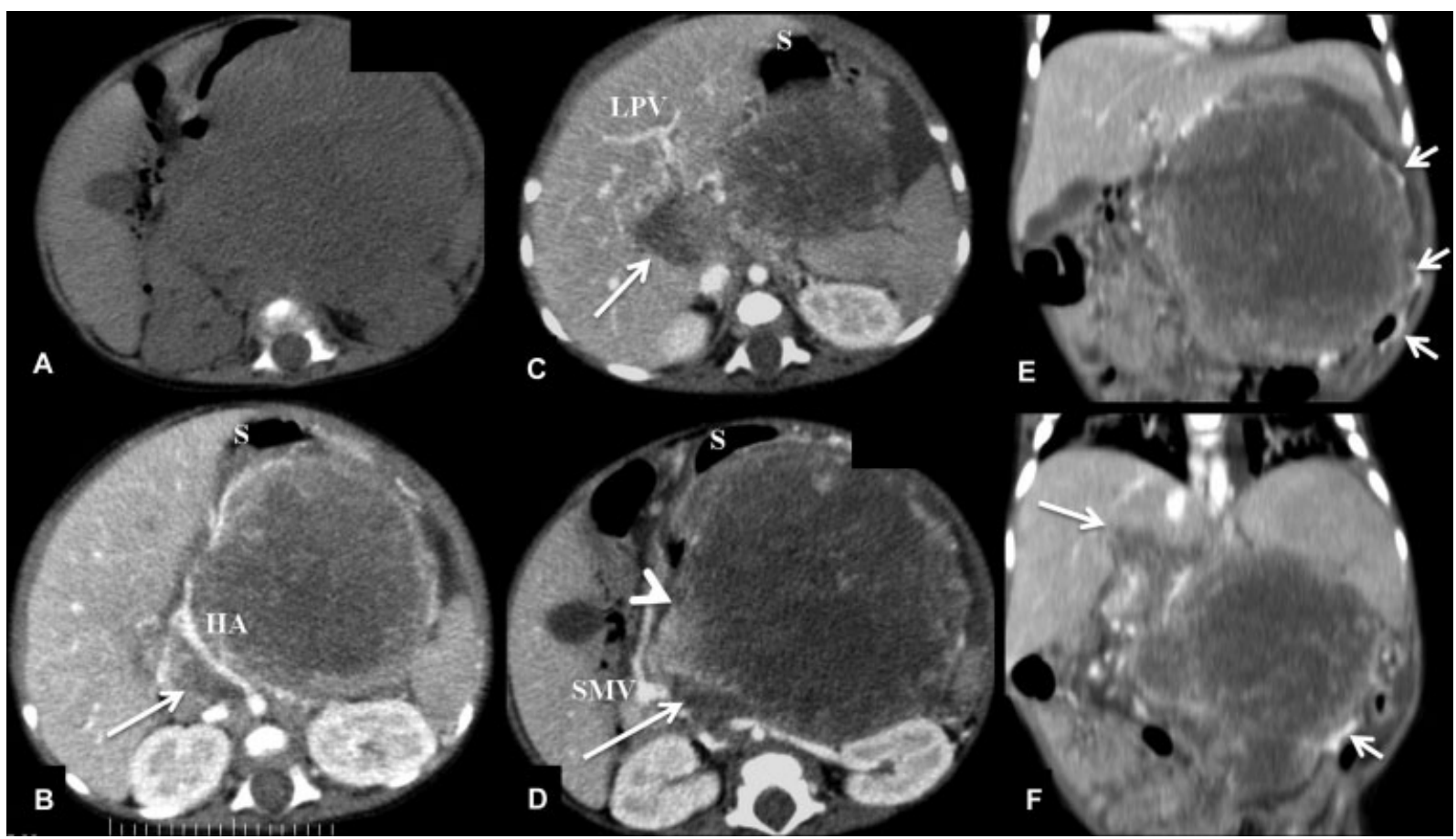

Fig. 4 Same case as - Fig. 3. NCCT (A) and CECT axial (B-D) and coronal (E, F) images reveal a solid mass with necrotic areas seen in the lesser sac region, displacing the stomach $(\mathrm{S})$ anteriorly. Common hepatic artery (HA) is draped around it. A claw sign is seen with the proximal body of pancreas (arrowhead in D), suggesting pancreatic origin. There is thrombosis of splenic vein and portal vein (long arrows), extending into proximal left portal vein (LPV). Multiple collaterals are seen (short arrows) reconstituting superior mesenteric vein (SMV). CECT, contrastenhanced computed tomography; NCCT, noncontrast computed tomography.

along with multiple venous collaterals. A small metastatic lesion was seen in segment 7 of the liver, along with few enlarged retroperitoneal lymph nodes. Imaging findings were consistent with a malignant mass of pancreatic origin, likely a pancreatoblastoma.

The detailed imaging findings of the three cases are summarized in - Table 1.

\section{Discussion}

An intravascular tumor thrombus results from tumor extension into the vessel. In adults, tumor thrombus in the portal vein is known to be associated with hepatic and pancreatic malignancies (most commonly hepatocellular carcinoma and pancreatic ductal carcinoma). On imaging, key features

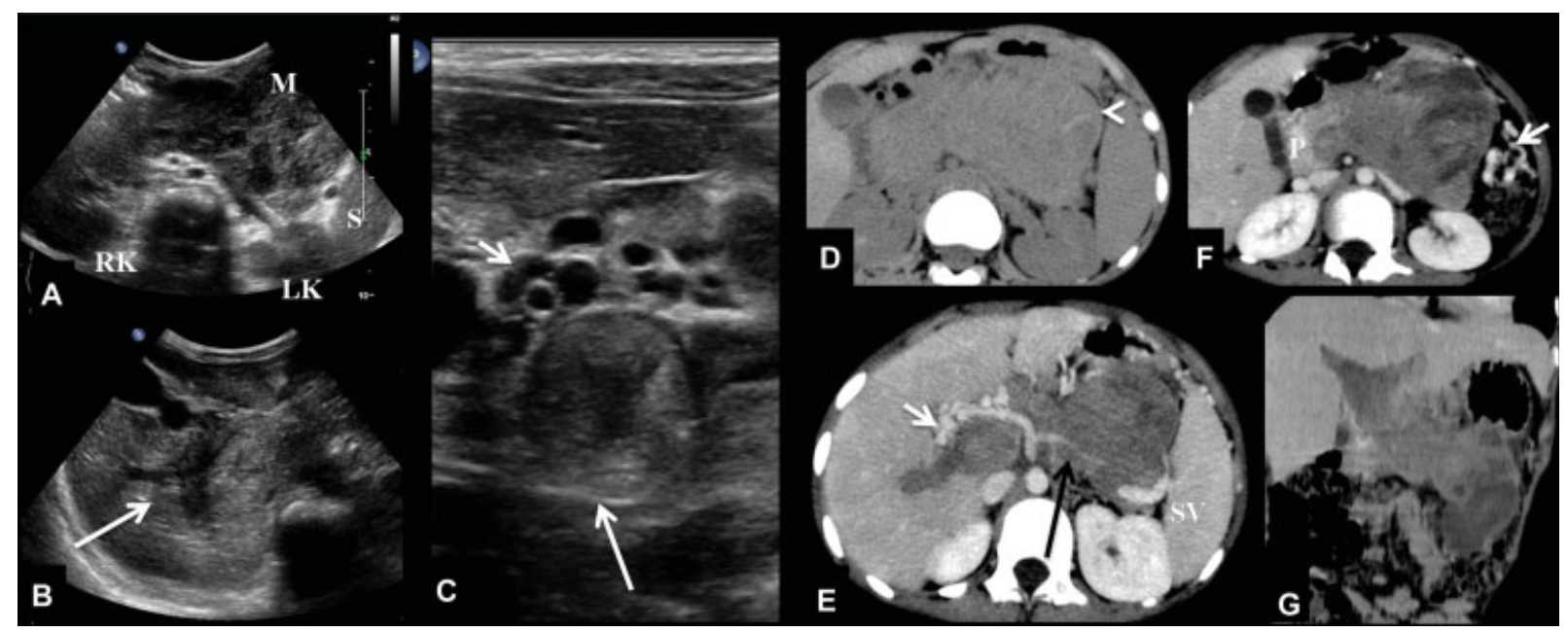

Fig. 5 An 8-year-old-boy with abdominal lump. USG (A-C) shows a large mass (M) in the region of distal body and tail of pancreas, with few cystic areas. Extensive tumor thrombus is evident within the intrahepatic portal vein branches and main portal vein (long arrows), with collaterals at porta (small arrow in C). NCCT (D) shows faint focal calcification (arrowhead) within the mass. CECT axial (E, F) and coronal MinIP (G) sections confirm a large mass arising from the pancreas (P), encasing the splenic artery (black arrow in $E$ ). Tumor thrombus expands the nonopacified splenic and portal veins, multiple collaterals (small arrows) are seen. A part of splenic vein (SV) is seen close to hilum. CECT, contrast-enhanced computed tomography; LK, left kidney; NCCT, noncontrast computed tomography; RK, right kidney; S, spleen; USG, ultrasonography. 
Table 1 Imaging findings in the three pancreatoblastoma cases

\begin{tabular}{|c|c|c|c|}
\hline & Case 1 & Case 2 & Case 3 \\
\hline Age/sex & 2 years/female & 7 months/male & 8 years/male \\
\hline Clinical presentation & $\begin{array}{l}\text { Pain abdomen, bleeding per } \\
\text { rectum } \times 5 \text { days. Lump palpable } \\
\text { on examination }\end{array}$ & Abdominal lump $\times 1$ month & $\begin{array}{l}\text { Abdominal lump, constipa- } \\
\text { tion for } 1 \text { month }\end{array}$ \\
\hline Alpha fetoprotein level & Not available & Elevated $(>2,000 \mathrm{ng} / \mathrm{mL})$ & Not available \\
\hline Tumor size & $\begin{array}{l}10 \mathrm{~cm} \times 7 \mathrm{~cm} \times 9.5 \mathrm{~cm} \\
(\mathrm{CC} \times \mathrm{TR} \times \mathrm{AP})\end{array}$ & $\begin{array}{l}9.5 \mathrm{~cm} \times 10 \mathrm{~cm} \times 10 \mathrm{~cm} \\
(\mathrm{CC} \times \mathrm{TR} \times \mathrm{AP})\end{array}$ & $\begin{array}{l}12 \mathrm{~cm} \times 10 \mathrm{~cm} \times 6 \mathrm{~cm} \\
(\mathrm{CC} \times \mathrm{TR} \times \mathrm{AP})\end{array}$ \\
\hline Origin & Pancreatic body and tail & Pancreatic body and tail & Pancreatic body and tail \\
\hline Echogenicity on USG & $\begin{array}{l}\text { Predominantly isohypoechoic to } \\
\text { liver, with small cystic areas }\end{array}$ & $\begin{array}{l}\text { Hyperechoic (compared with } \\
\text { liver) }\end{array}$ & $\begin{array}{l}\text { Heterogeneously } \\
\text { hyperechoic }\end{array}$ \\
\hline $\begin{array}{l}\text { CT enhancement } \\
\text { characteristics }\end{array}$ & $\begin{array}{l}\text { Mild, heterogeneous enhance- } \\
\text { ment with multiple, small non- } \\
\text { enhancing areas }\end{array}$ & $\begin{array}{l}\text { Heterogeneous enhance- } \\
\text { ment with large nonenhanc- } \\
\text { ing areas }\end{array}$ & $\begin{array}{l}\text { Mild, heterogeneous en- } \\
\text { hancement with few small } \\
\text { non-enhancing areas }\end{array}$ \\
\hline Calcification & Absent & Absent & Present \\
\hline Necrosis & Present & Present & Present \\
\hline Arterial encasement & Absent & Absent & $\begin{array}{l}\text { Present - involving splenic } \\
\text { artery }\end{array}$ \\
\hline Venous invasion & $\begin{array}{l}\text { Present involving splenic vein, } \\
\text { MPV, RPV, proximal LPV }\end{array}$ & $\begin{array}{l}\text { Present involving splenic } \\
\text { vein, MPV, RPV, proximal LPV }\end{array}$ & $\begin{array}{l}\text { Present involving splenic } \\
\text { vein, SMV, MPV, RPV, LPV }\end{array}$ \\
\hline Venous collaterals & Present & Present & Present \\
\hline $\begin{array}{l}\text { Biliary/pancreatic } \\
\text { ductal dilatation }\end{array}$ & $\begin{array}{l}\text { Present: mild dilatation of pe- } \\
\text { ripheral intrahepatic biliary } \\
\text { radicles }\end{array}$ & Absent & Absent \\
\hline Ascites & Absent & Present & Present-minimal \\
\hline $\begin{array}{l}\text { Retroperitoneal } \\
\text { lymphadenopathy }\end{array}$ & Absent & Absent & Present \\
\hline Features of local invasion & $\begin{array}{l}\text { Loss of fat planes with splenic } \\
\text { flexure of colon }\end{array}$ & $\begin{array}{l}\text { Loss of fat planes with } \\
\text { greater curvature of stomach } \\
\text { and liver, porta hepatis }\end{array}$ & $\begin{array}{l}\text { Loss of fat planes with } \\
\text { greater curvature of stomach } \\
\text { and first part of duodenum }\end{array}$ \\
\hline Liver metastases & Absent & Absent & Present \\
\hline Any other finding & $\begin{array}{l}\text { Few venous collaterals around } \\
\text { the rectum and sigmoid colon }\end{array}$ & Small left pleural effusion & Mild splenomegaly \\
\hline
\end{tabular}

Abbreviations: AP, anteroposterior; CC, craniocaudal; LPV, left portal vein; MPV, main portal vein; RPV, right portal vein; SMV, superior mesenteric vein; TR, transverse.

that help in diagnosis of tumor thrombus (and its differentiation from bland thrombus) include enhancement within the thrombus, its contiguity with the tumor mass, and expansion of the involved vein. ${ }^{3}$ The CT density and appearance of the thrombus matches that of the tumor mass.

Pancreatoblastomas are rare tumors. They usually occur in the first decade of life, and a male preponderance is seen. Of our cases, two were boys: one infant and the other was 8-years of age. The aggressive nature of these lesions is reflected in their rapid growth. Patients usually present with an abdominal lump, often large at diagnosis. Congenital cases can occur, often associated with Beckwith-Wiedemann syndrome (a congenital overgrowth disorder characterized by hemihypertrophy, macroglossia, organomegaly with increased risk of malignancies including Wilm's tumor, hepatoblastoma, adrenocortical carcinoma, and pancreatoblastoma). These congenital tumors are predominantly cystic. ${ }^{2}$ Elevated levels of $\alpha$-fetoprotein (AFP) may be seen in at least one-third of cases of pancreatoblastoma. Among our cases, AFP levels were estimated only in one case, and found significantly elevated.

On imaging, pancreatoblastomas may appear as circumscribed solid masses, though larger lesions are typically less well defined. They appear heterogeneously hyperechoic on sonography and may show cystic (necrotic) areas within. CT or magnetic resonance imaging (MRI) may demonstrate a heterogeneously enhancing mass arising from the pancreas, often protruding into the lesser sac. The large size can make it difficult to ascertain the organ of origin on imaging studies. ${ }^{1,2,4}$ In our cases, the pancreatic origin of the masses was evident on ultrasonography only in one case which was an older child ( 8 years of age). In the other two cases, the organ of origin was not clear on USG; however, CT was able to demonstrate origin from the pancreas. 
Pancreatoblastomas may show calcification as was seen in one of our cases. They do not usually cause significant dilatation of the pancreatic duct (PD) or the common bile duct (CBD). This is attributed to the relatively soft consistency of the tumors. Sometimes, large tumors in the pancreatic head may show associated biliary dilatation. All three of our cases were large masses arising from the pancreatic body and tail, and therefore showed no dilatation of $\mathrm{PD} / \mathrm{CBD}$. Large tumors often show arterial encasement, and may show invasion of surrounding structures. Metastases occur most commonly to the liver and abdominal lymph nodes. ${ }^{1,2}$

The heterogeneity on imaging results from presence of tumoral necrosis and hemorrhage. Sometimes, assessment of enhancement on CT may be difficult. Limited noncontrast scans may be done before doing CECT, this helps in accurate quantification of enhancement. Previously, authors have described that presence of areas within the tumor that isoattenuating compared with adjacent enhanced vessels (on CECT) is helpful in detection of contrast enhancement. ${ }^{4}$ Such areas were seen in one of our cases (case 2).

Venous invasion into the splenoportal axis and portal vein by pancreatoblastoma has been previously described as rare. ${ }^{2,5,6}$ However, this was seen in all three of our cases, all of whom showed extensive tumor thrombosis involving the retropancreatic splenic vein and extending into the portal vein and its intrahepatic branches. Expansion of the involved veins was evident, along with multiple venous collaterals surrounding the tumor. The diagnosis of tumor thrombus can be made on imaging by virtue of the thrombus showing matching echogenicity to the mass on USG, and similar density and enhancement characteristics on CT.

These cases highlight that portal vein thrombosis in pancreatoblastoma is an important imaging finding which can suggest the correct diagnosis, especially in large tumors when the organ of origin is difficult to make out. The presence of venous collaterals on CECT/MRI is a valuable indirect sign of venous thrombosis, and should prompt a careful evaluation of the portal venous system. The involvement of the intrahepatic branches of the portal vein may sometimes be misinterpreted as liver metastases; therefore it is important to trace the portal vein and splenoportal axis in its entirety to differentiate between the two.

The imaging differential diagnosis may include other tumors arising from the pancreas, as well as other large abdominal masses. Other tumors arising from the pancreas are rare in children. Solid papillary epithelial neoplasm (SPEN) or solid pseudopapillary tumor is a solid-cystic tumor which most often involves the pancreatic head, commonly occurs in adolescents, and is more common in girls. Imaging features are those of a mixed solid-cystic tumor, with hemorrhage/blood products in more than half the cases (the best appreciated on MRI). Calcification may be seen in one-third of cases. The tumor has low malignant potential, and metastases are uncommon at presentation. ${ }^{1,2}$

Islet cell tumors of the pancreas, though uncommon in children, may occur, especially in association with multiple endocrine neoplasia syndromes. These tumors may be func- tional or nonfunctional. The most typical imaging feature is hypervascularity on arterial phase imaging. ${ }^{1}$

Nonepithelial tumors of pancreas include lymphoma, usually as a part of disseminated non-Hodgkin's or Burkitt's lymphoma. Imaging may reveal one or more homogeneous pancreatic masses, usually without associated pancreatic ductal or biliary dilatation. ${ }^{1,2}$

More common causes of large malignant abdominal masses in children are Wilm's tumor, neuroblastoma, lymphoma, and hepatoblastoma. In children with Wilm's tumor, imaging will demonstrate a circumscribed mass of renal origin which may show renal vein invasion but does not show encasement of vessels. Neuroblastoma masses may be adrenal or extra-adrenal in origin, often show calcification and vascular encasement, and may show intraspinal extension. Lymphoma masses may be large, usually homogeneous, and may involve solid organs but almost always in association with bulky lymph nodal masses. Hepatoblastoma is the most common tumor of hepatic origin in the first decade of life and may be considered in the imaging differential diagnosis of pancreatoblastoma. Elevated levels of AFP are seen in up to $90 \%$ cases of hepatoblastoma and about onethird cases of pancreatoblastoma. Hepatoblastomas are usually well-circumscribed, solid tumor masses, often hypervascular, and may show calcification on CT. Demonstration of origin of the mass from the liver is the most reliable means of differentiation from pancreatoblastoma.

Other tumors which may show portal vein invasion include hepatoblastomas and hepatocellular carcinomas. Both these tumors tend to involve the portal vein and its intrahepatic branches, and usually spare the splenic vein. Venous invasion into the renal/adrenal veins and inferior vena cava may be seen in Wilm's tumor and adrenocortical carcinoma.

\section{Conclusion}

To conclude, pancreatoblastomas, though rare, are the commonest pancreatic masses in children in the first decade of life and should be considered in the differential diagnosis of large upper abdominal masses in this age group. Tumor thrombus within the splenic vein and portal vein is a useful ancillary imaging feature of these tumors and may help to clinch the diagnosis.

Conflicts of Interest

None declared.

\section{References}

1 Shet NS, Cole BL, Iyer RS. Imaging of pediatric pancreatic neoplasms with radiologic-histopathologic correlation. AJR Am J Roentgenol 2014;202(06):1337-1348

2 Chung EM, Travis MD, Conran RM. Pancreatic tumors in children: radiologic-pathologic correlation. Radiographics 2006;26(04): 1211-1238

3 Quencer KB, Friedman T, Sheth R, Oklu R. Tumor thrombus: incidence, imaging, prognosis and treatment. Cardiovasc Diagn Ther 2017;7(Suppl 3):S165-S177 
4 Montemarano H, Lonergan GJ, Bulas DI, Selby DM. Pancreatoblastoma: imaging findings in 10 patients and review of the literature. Radiology 2000;214(02):476-482

5 Gupta AK, Mitra DK, Berry M, Dinda AK, Bhatnagar V. Sonography and CT of pancreatoblastoma in children. AJR Am J Roentgenol 2000;174(06):1639-1641
6 Ibuka S, Uehara S, Ueno T, et al. Complete resection of pancreatoblastoma with portal vein obstruction after high-dose chemotherapy: a case report. J Pediatr Hematol Oncol 2017;39(05): e275-e278 